\title{
El uso de la teoría de redes sociales en la representación y análisis de textos. De las redes semánticas al análisis de redes textuales
}

\author{
JoAN MiQuel Verd PERICÁS \\ Universidad Autónoma de Barcelona \\ JoanMiquel.Verd@uab.es
}

Recepción: septiembre 2005

Aceptación: noviembre 2005

\section{INTRODUCCIÓN}

La idea de red se aplica hoy en día a multitud de fenómenos. Entre las ciencias sociales - aunque no únicamente- ha abierto nuevos horizontes de explicación y comprensión de los objetos que les son propios, y pocos fenómenos se escapan a la posibilidad de ser abordados poniendo en juego el componente relacional. Las metodologías de análisis de textos también se han dejado conquistar por esta tendencia, aunque con fecha relativamente muy reciente. A pesar de que la idea de «red de significados» parece casi intrínseca a la comprensión de un texto, la gran mayoría de aplicaciones de los instrumentos conceptuales y metodológicos del análisis de redes sociales a datos de carácter textual tiene menos de quince años.

Esta juventud en la aplicación de esta perspectiva al análisis de textos tiene consecuencias previsibles. Los términos con que se designan los procedimientos de análisis son variadísimos y no suelen ayudar a identificar puntos de contacto entre orientaciones parecidas. No obstante, es cierto que en los últimos cinco años van surgiendo lentamente trabajos publicados en inglés que introducen una cierta sistematización. El presente artículo pretende hacer lo mismo en el ámbito de la lengua española. Se abordan en él, por tanto, todas aquellas aproximaciones metodológicas a la representación y análisis de textos que en mayor o menor medida se inspiran en la teoría de redes sociales. El artículo no pretende una absoluta exhaustividad en la revisión de todos los trabajos publicados con esta orientación, pero sí aspira a no dejar de lado ninguno de los modos en que la teoría de redes sociales ha sido utilizada en el análisis de textos. No se abordan, por otro lado, todas aquellas aproximaciones que pese a utilizar la idea de red o 
relación' no contemplan un uso explícito ni de la representación ni del análisis mediante redes.

El artículo se divide en tres partes principales y un último punto de conclusiones. En la primera parte se establecen los antecedentes de la aplicación de redes al análisis de textos. Estos antecedentes se sitúan en el uso de grafos en la representación de redes semánticas o mapas cognitivos. Este uso ha recibido un renovado impulso en años recientes y es habitual hoy en día en gran número de análisis cualitativos. La segunda y tercera parte se dedican respectivamente a lo que hemos denominado análisis de redes de palabras y análisis de redes textuales. La diferencia fundamental entre unas aproximaciones y otras es que en las primeras las redes son el resultado de la representación de la proximidad entre las palabras o conceptos que aparecen en un texto, mientras que en las segundas el tipo de relaciones representadas tienen una gran variedad y son fruto de la interpretación humana. En el primer tipo de orientaciones son las palabras las que tienen el protagonismo en las redes, en el segundo tipo de orientaciones el protagonismo es compartido entre palabras y relaciones. Finalmente, en las conclusiones se presentan los aspectos más relevantes abordados en el artículo y unas reflexiones finales relativas a los posibles desarrollos futuros de todo el conjunto de aplicaciones reseñadas.

\section{LAS REDES SEMÁNTICAS COMO MODO DE REPRESENTACIÓN DEL CONOCIMIENTO}

El uso de grafos para la representación del conocimiento que posee una persona, una institución o un colectivo ha sido habitual desde tiempos antiguos. Este tipo de aplicaciones puede considerarse, por tanto, el primer uso de las redes en la representación de relaciones entre términos linguísticos (en este caso, conceptos). Según Sowa (1991) fue el filósofo neoplatónico Porfirio el primero en utilizar grafos en este campo, que posteriormente se asentó con los trabajos de Peirce a finales del siglo XIX, y con los del psicólogo Selz a principios del XX. El término más habitual para designar este tipo de representaciones es el de redes semánticas, aunque los términos aplicados a estas representaciones son variadísimos. Sirva como ejemplo de esta pluralidad terminológica la siguiente afirmación de Richards y Richards (1994:458): «Diagramas de conceptos, grafos conceptuales, redes semánticas y redes conceptuales son (aproximadamente) diferentes nombres dados a la misma idea, la de representar información de carácter conceptual de forma gráfica».

Puede definirse una red semántica como una representación gráfica en forma de nodos y arcos interconectados (es decir, en forma de red) que tiene como ob-

1 Puede encontrarse una revisión de todo este conjunto de aproximaciones en Lozares et al. (2003). El artículo, además, pone de manifiesto, precisamente, los puntos de contacto y las diferencias entre las aproximaciones «relacionales» y las «reticulares». 
jetivo representar un determinado conocimiento. El desarrollo que en los últimos años han experimentado las ciencias cognitivas aplicadas a la informática y a la inteligencia artificial ha dado un fuerte impulso a las redes semánticas entendidas como sistemas lógicos formales, aunque con anterioridad habían sido ya usadas en ciencias como la filosofía, la linguística y la psicología.

En la Figura 1 puede apreciarse un ejemplo muy sencillo de red semántica. Se trata concretamente de una jerarquía de tipos, en que los nodos representan conceptos y los lazos representan una pertenencia taxonómica ${ }^{2}$.

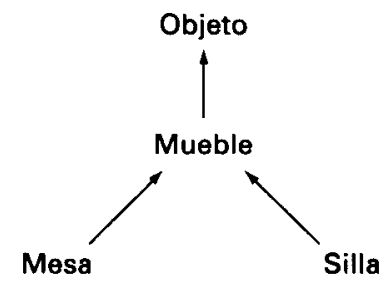

Fuente: Elaboración propia.

Figura 1. Ejemplo de jerarquía de tipos (red semántica de carácter taxonómico).

Como se ha señalado, las redes semánticas no surgen inicialmente como instrumento de análisis y/o representación de textos, sin embargo constituyen el antecedente más inmediato de los análisis que a partir de mediados de los años 80 han utilizado los instrumentos del análisis de redes sociales como modo de analizar datos de carácter textual. De hecho, estos desarrollos no sólo toman la idea de representación gráfica en forma de red para realizar un tratamiento próximo al análisis de redes sociales, sino que en algunos casos denominan a las representaciones realizadas con el término de red semántica (véanse Doerfel 1998, Jang y Barnett 1994, Popping 2003, Schnegg y Bernard 1996).

No obstante, antes de abordar los análisis que propiamente utilizan la metodología y conceptos de redes sociales en el análisis de textos es interesante observar los usos que dentro de las técnicas cualitativas de análisis se han hecho (y se hacen) de las redes semánticas. Estos usos se benefician especialmente de la fortaleza descriptiva de las representaciones mediante grafos y de su capacidad para explorar relaciones entre conceptos. De hecho, estos usos pueden dividirse en dos, que no son mutuamente excluyentes.

El primero de estos usos se ha destinado a mostrar las representaciones y esquemas cognitivos de las personas investigadas. Según Krippendorf (2004:293) el origen de este tipo de redes se encuentra posiblemente en las investigaciones de la antropología cultural que deseaban estudiar las estructuras cognitivas y los modelos mentales de las poblaciones estudiadas. Sus aplicaciones actuales son

${ }^{2}$ Este tipo de representaciones han sido desarrolladas principalmente para establecer el significado de las palabras o conceptos representados (véase Johnson-Laird 1990: cap. 18). 
diversas, no sólo en el estudio de colectividades, sino también para conocer con detalle el marco cognitivo de las personas que, por ejemplo, va a ser entrevistadas o participarán en un grupo de discusión. Miles y Huberman (1994:132137) distinguen en este terreno entre las taxonomías en forma de familia (folk taxonomies), que tienen una estructura jerárquica, y los mapas cognitivos (cognitive maps), que no se organizan jerárquicamente.

El segundo de estos usos - probablemente el más extendido- tiene como objetivo representar y explorar las estructuras o esquemas conceptuales que están en la base o son el resultado de un determinado proceso de investigación. No se trata por lo tanto de representar el conocimiento de las personas investigadas, sino el conocimiento que aplica o «descubre» el investigador o investigadora. Miles y Huberman ha sido unos de los responsables de la popularización de este tipo de redes en los análisis de carácter cualitativo y defienden (1994:151-165 y 228-238) su utilidad especialmente como herramienta para explorar las relaciones de causalidad entre los conceptos manejados en una investigación (variables dependientes $\mathrm{e}$ independientes). La fortaleza de estas representaciones para abordar relaciones complejas ha llevado a su aplicación en la exploración de propiedades de carácter lógico (transitividad, simetricidad) y también en la dimensionalización conceptual, en lo que se ha denominado codificación de segundo orden (Prein et al. 1995).

La utilidad de las redes semánticas como instrumento en el proceso de investigación ha llevado a algunos programas de análisis cualitativo asistido por ordenador a incorporar herramientas de representación y (en menor medida) gestión de redes. De estos programas, ATLAS.ti y Nud*ist son los más conocidos, aunque el primero permite una mayor flexibilidad en el uso de las redes. Debe mencionarse que en estos dos programas las redes semánticas utilizadas no representan relaciones entre los términos de los documentos analizados, sino entre los conceptos (con un nivel menor o mayor de abstracción) que van siendo aplicados a medida que se desarrolla el análisis ${ }^{3}$. En la Figura 2 se muestra una red semántica elaborada con el programa ATLAS.ti que recoge las relaciones obtenidas en un análisis entre cuatro códigos diferentes.

Debe destacarse que este tipo de representaciones no pueden considerarse estrictamente una aplicación de la perspectiva de redes sociales al análisis de textos (o más generalmente a los análisis cualitativos). Lonkila y Harmo (1999:56), refiriéndose concretamente al programa ATLAS.ti, exponen sucintamente por qué: «En primer lugar, carece[n] de las líneas básicas para el cálculo de índices estructurales de la red. En segundo lugar, no acepta[n] redes en su forma matricial». Ello reduce el uso de las redes a instrumentos de (re)presentación. De todos modos, y tal como veremos más adelante, los análisis que sí utilizan los con-

${ }^{3}$ Ambos programas se inspiran ampliamente en los procedimientos de la Grounded Theory (Glaser y Strauss 1967), cuya apuesta por una codificación con diferentes niveles de abstracción y por la exploración de relaciones entre categorías alienta claramente el uso de mecanismos de representación gráfica. 


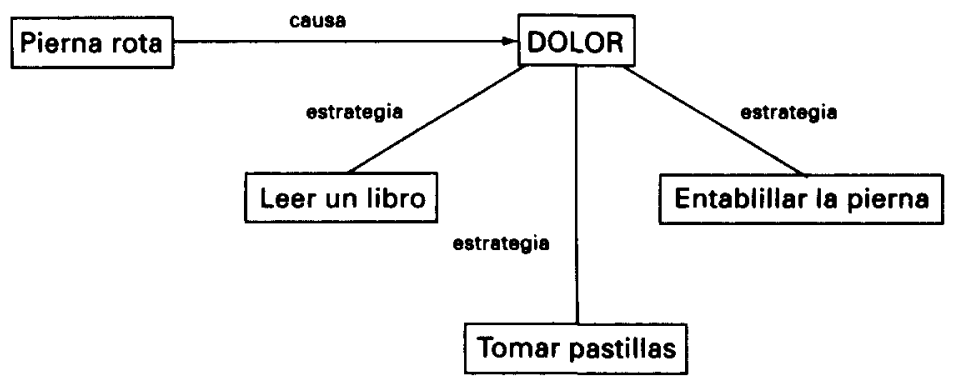

Fuente: Adaptación a partir de las Figuras 85 y 87 de Muhr y Friese (2004:214 y 217).

Figura 2. Red semántica realizada con ATLAS.ti que representa los vínculos entre cuatro códigos utilizando las relaciones causa y estrategia.

ceptos e indicadores propios del análisis de redes sociales tienen numerosos puntos de contacto con los usos expuestos hasta el momento.

\section{ANÁLISIS DE REDES DE PALABRAS}

Las primeras aproximaciones que utilizaron los instrumentos de la teoría de redes sociales para analizar matrices de palabras por palabras no tenían como objetivo fundamental el análisis de textos. Todo un conjunto de autores provenientes del campo del análisis de redes de comunicación ${ }^{4}$ empezaron a partir de la segunda mitad de los años 80 a construir redes en que los nodos estaban constituidos por los términos lingüísticos compartidos por un conjunto de actores. Las redes construidas en este conjunto de trabajos se caracterizan por establecer redes de palabras en que la relación representada en los arcos es la «copresencia». Esta copresencia puede estar basada en el hecho de "compartir» un mismo emisor (es decir la relación se establece si un mismo actor utiliza ambos términos) o en compartir un mismo texto o una parte limitada de un mismo texto. Generalmente, uno de los objetivos habituales de estas redes de palabras es conocer cuáles son los conceptos comunes dentro de un conjunto dado de actores; es por ello que en algunos casos estas representaciones se denominan también redes cognitivas o redes semánticas (Doerfel 1998), posiblemente recordándonos con el término que lo que se está haciendo es desarrollar y/o adaptar las técnicas de representación cognitiva ya existentes. Con el tiempo, este tipo de redes de palabras se han aplicado a otros objetivos. Se utilizan como modo de representación de un texto o conjunto de textos, sin que necesariamente quieran establecerse ulteriores inferencias en relación al productor o productores de los documentos analizados. En la Figura 3 se utiliza, a modo de ejemplo, el primer

${ }^{4}$ Se trata de redes de actores (personas, instituciones, etc.) en que los arcos representan quién se comunica con quién. 
A una nariz

Érase un hombre a una nariz pegado, Érase una nariz superlativa, Érase una nariz sayón y escriba, Érase una alquitara pensativa [...]

Fuente: Elaboración propia.

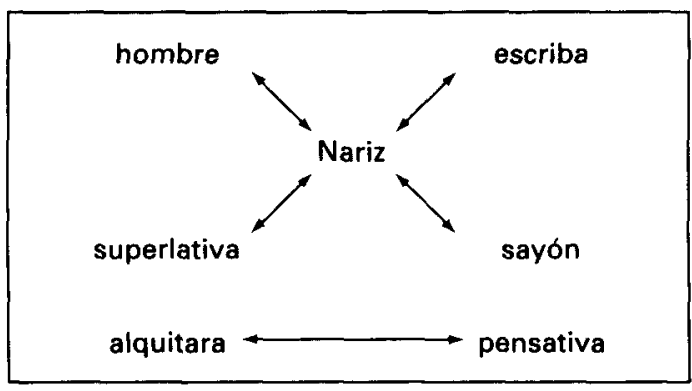

Figura 3. Representación gráfica de la relación "copresencia en la misma línea» entre los nombres sustantivos y adjetivos del primer cuarteto del soneto de Francisco de Quevedo A una nariz.

cuarteto de un soneto de Quevedo para representar una red de palabras extremadamente sencilla en que se relacionan dos palabras si están presentes en una misma línea.

En la construcción de redes de palabras pueden observarse dos procedimientos principales. En primer lugar, aquellas que se construyen sobre la base de una matriz de actores por palabras (matriz de afiliación), de la que puede obtenerse posteriormente una matriz valorada de palabras por palabras (matriz de adyacencia) que representa el número de ocasiones en que cada una de ellas coocurre en un mismo actor. Existen diferentes formas de obtener estas relaciones entre actores y palabras (cuestionario cerrado, grupo de discusión, entrevista en profundidad, elección a partir de una lista...) ${ }^{5}$, sin que necesariamente tenga que realizarse un análisis de los textos producidos por los actores. En segundo lugar, otro modo de construcción de redes de palabras es la utilización de algún método de codificación automatizada que tenga en cuenta las coocurrencias de unas mismas palabras o conceptos en un espacio textual de una amplitud dada dentro del texto o conjunto de textos analizados. En este caso la relación que une las palabras es la copresencia en la «ventana» de texto considerada.

Debe destacarse que todas estas aproximaciones al análisis de textos que aquí calificamos como análisis de redes de palabras tienen importantes puntos de contacto con el análisis de contenido cuantitativo de corte clásico (Bauer 2000, proporciona una buena evaluación). Se trata en definitiva de procedimientos que se fundamentan en la codificación automatizada de las palabras o lexemas presentes en un texto (lo cual exige, como en el análisis de contenidos, una depuración previa de las palabras con escaso valor semántico) a las que se somete a un tratamiento basado en el análisis de redes sociales.

Esta similitud, también está en el origen de las críticas que han recibido estas aproximaciones. Carley ha criticado este tipo de análisis (que denomina

\footnotetext{
5 Wuehrer (2001) realiza una buena síntesis de las diferentes técnicas aplicables.
} 
proximity analysis) por su incapacidad para recoger el diferente sentido que otorga a un enunciado la alteración del orden de sus términos (1993:105). También Van Meter realiza un cierto cuestionamiento de la bondad de la codificación automatizada; su propuesta (1999:78) es la de organizar las palabras contabilizadas automáticamente en categorías más generales, de modo que puedan recogerse de modo más preciso los sentidos presentes en los textos analizados y después aplicar los procedimientos de análisis de redes. En términos generales, puede afirmarse que aquello de que adolecen este tipo de análisis es precisamente de una aplicación profunda de todas las posibilidades que ofrece el análisis de redes sociales. Las redes son utilizadas principalmente como instrumento de representación gráfica y por su versatilidad en el cálculo matricial, utilizándose excepcionalmente la posibilidad de asignar un contenido sustantivo a las relaciones entre palabras. De hecho, se codifica un tipo de relación que es simplemente «coincidir/estar cercano» en el texto, lo cual implica un escaso uso de la posibilidad de caracterizar (en términos de intensidad, signo, dirección o significado) las relaciones entre las unidades (nodos) de la red textual resultante. Esta es la razón por la cual Popping (2000) no considera el análisis de redes de palabras (word-network analysis) como una verdadera aplicación de la perspectiva de redes sociales al análisis de textos, argumentando que tiene carácter temático, basado exclusivamente en la coocurrencia de términos.

Con todo, deben reconocerse como ventajas de estas aproximaciones la simplicidad de los procedimientos y la capacidad insuperable de analizar grandes cantidades de texto. Describimos a continuación con cierto detalle algunos de los trabajos encuadrados en esta orientación, siguiendo un orden que va desde los más característicos dentro de este conjunto de aproximaciones a aquellos que suponen variantes más alejadas.

Sin duda los trabajos de Danowski $(1988,1993)$ son los que mejor ilustran el procedimiento de análisis basado en redes de palabras creadas mediante el uso de la codificación automatizada. Incluso el término que utiliza para denominar su procedimiento (word-network analysis) se ha extendido más allá de sus trabajos para denominar todo el conjunto de aproximaciones con bases similares. Estos trabajos, así como los de Freeman y Barnett (1994), Schnegg (1997) o Jang y Barnett (1994) se sitúan en el marco de las investigaciones sobre cultura organizativa, con el objetivo de ofrecer un «método menos subjetivo y más preciso» (Freeman y Barnett 1994:62).

El procedimiento (Danowski 1993:203-15) consiste en crear una matriz de palabras por palabras a partir de la coocurrencia en una "ventana corredera», cuya amplitud varía entre los trabajos (entre 7 y 11 palabras para Danowski 1993; entre 3 y 10 palabras para Freeman y Barnett 1994;5 palabras para Schnegg 1997). El origen de los datos puede ser muy variado: mensajes electrónicos entre miembros de una misma empresa, noticias aparecidas en los periódicos, transcripciones de entrevistas y grupos de discusión, fragmentos de libros...

También las problemáticas concretas tratadas son variadas. Danowski (1988) analiza las transcripciones de grupos de discusión formados por directivos de 
compañías sometidos a traslado; Freeman y Barnett (1994) intentan identificar cuáles son las características del lenguaje utilizado en los mensajes escritos dirigidos a los empleados en una empresa fabricante de material médico; y Jang y Barnett (1994) y Schnegg (1997) se preocupan por las diferencias culturales entre 35 empresas norteamericanas y japonesas a partir del análisis de las palabras más utilizadas en una selección de informes públicos.

El resultado es siempre una red de palabras que puede analizarse de diferentes modos. Mientras Danowski se orienta principalmente hacia un análisis estructural, en el sentido de que normalmente presta más atención a los grupos de palabras que se forman y a las distancias existentes entre palabras que a la posición concreta de ciertas palabras en relación a las otras (1988:412-17); Schnegg (1997) se preocupa especialmente por la «comunidad semántica» (redes egocentradas) de las palabras más utilizadas en cada grupo de empresas, investigando también a través de qué palabras se produce la relación entre las diferentes comunidades semánticas (es decir, entre los términos más utilizados por cada grupo de empresas).

Una variación respecto a las redes construidas mediante «ventanas correderas» puede encontrarse en los trabajos de Van Meter (Van Meter y Turner 1997, Van Meter 1999). En este caso la coocurrencia de palabras viene determinada por la presencia simultánea en el mismo texto; es decir el marco en el que se contabiliza la coocurrencia son las unidades textuales completas. Previamente se define el tipo de documento y el carácter de las palabras que se desean analizar, «sean éstas nombres geográficos en las biografías oficiales soviéticas, palabras clave en los abstracts de artículos científicos, o términos en la patente de un invento" (Van Meter y Turner 1997:36) y a continuación se procede a la construcción y análisis de las redes.

Van Meter y Turner denominan cognitive mapping a la aproximación que realizan, cuyo objetivo principal es detectar, a partir de los conceptos principales utilizados dentro de un determinada disciplina académica, diferentes grupos de investigadores, así como la propia evolución de la disciplina. Concretamente, estos autores han analizado las coocurrencias de palabras clave en los resúmenes cortos aparecidos en las reseñas de Sociological Abstracts relacionadas con los temas del SIDA (Van Meter y Turner 1997) y el capital social (Van Meter 1999). Utilizando combinadamente los programas Lexinet y Leximappe se identifican las coocurrencias de palabras clave, a continuación se calcula un índice de proximidad -basado, obviamente, en el número de coocurrencias-entre ellas, y a continuación se utiliza este índice para construir conglomerados de palabras con un máximo de diez por grupo. Estos grupos se representan posteriormente sobre dos ejes, siendo el primero un indicador de centralidad (la media de los vínculos entre el conglomerado y las palabras fuera de él), y el segundo un indicador de densidad o cohesión interna del grupo (la media de la intensidad de asociación entre las unidades del conglomerado). La construcción de grupos y el cálculo de los indicadores de centralidad y densidad de cada uno de ellos permite a Van Meter y Turner describir la evolución de los contenidos de la actividad 
científica en que están interesados, así como las interrelaciones existentes entre los diferentes temas ${ }^{6}$.

También los trabajos realizados por el equipo formado en torno a Corman (Brandes y Corman 2003, Corman y Dooley 2001, Corman et al. 2002) constituyen un distanciamiento respecto al modelo de «ventanas correderas». El tipo de análisis textual que realizan, que denominan Centering Resonance Analysis (CRA) es posiblemente el más sofisticado de los análisis basados en la coocurrencia de palabras. Basándose en la teoría linguiística de los «centros conversacionales» (centering theory) el objetivo principal de este tipo de análisis es el de localizar las palabras con una posición estructural más relevante en un conjunto dado de textos (Corman et al. 2002:173). Para ello separan en primer lugar el texto en frases, frases que a su vez se descomponen, si es pertinente, en diferentes sintagmas nominales (noun phrases). Son los nombres y adjetivos presentes en los sintagmas nominales los que van a constituir los nodos de la red, siendo la relación que los va a unir el hecho de formar parte del mismo sintagma nominal o de sintagmas nominales consecutivos en la misma frase. La red de palabras resultante contiene diferentes intensidades en las relaciones, en función del número de veces que coocurren las palabras. El valor estructural de las palabras se obtiene mediante el cálculo de la centralidad de intermediación (betweennes centrality). Esta aproximación se ha aplicado a diferentes tipos de textos: por un lado al análisis de las informaciones que tras los atentados del $11 \mathrm{de}$ septiembre de 2001 estuvo difundiendo la agencia de noticias Reuters (Corman y Dooley 2001), por otro al análisis de transcripciones de interacciones lingüisticas entre dos grupos enfrentados (Corman et al. 2002), y por otro a la evaluación de las percepciones que un grupo de estudiantes tenían de dos textos diferentes (Corman et al. 2002). Así mismo se ha desarrollado una versión del análisis que pretende realizar una aproximación dinámica al desarrollo de los discursos (Brandes y Corman 2003).

Finalmente, como muestra del procedimiento que construye las relaciones entre palabras basándose en la copresencia en un mismo emisor mencionamos aquí el trabajo de Schnegg y Bernard (1996). El citado trabajo tenía como objetivo conocer qué conceptos representaban mejor las motivaciones centrales de un conjunto de estudiantes de antropología para elegir dicha titulación. Los autores analizaron cuantitativamente las transcripciones de 21 entrevistas, dando lugar a un listado de 261 palabras que, una vez depurado, quedó en 141 palabras representativas de diferentes «temas». No obstante, con el objetivo de reducir aún más este listado se confió a tres codificadores diferentes la elección de las palabras más representativas de acuerdo con el objetivo de la investigación, obteniéndose como resultado 49 palabras. El análisis se realizó sobre la matriz de $49 \times 49$

\footnotetext{
- No obstante, solamente se obtiene una distribución estructurada de los datos en el.caso de las investigaciones sobre el SIDA. En el caso de las investigaciones sobre el capital social el autor se ve obligado a realizar un tratamiento más tradicional basado en tablas de contingencia y en el agrupamiento de carácter «interpretativo» (sic).
}

EMPIRIA. Revista de Metodologfa de Ciencias Sociales. N. ${ }^{\circ}$ 10, julio-diciembre, 2005. pp. 129-150. ISSN: $1139-5737$ 
palabras, en que para cada par de palabras existía una puntuación representativa del número de veces que eran mencionadas conjuntamente por los informantes. Con el programa UCINET se fueron aplicando sucesivamente diferentes valores de corte para discriminar aquellas con menor presencia en los textos de aquellas mayoritariamente utilizadas; de este modo se fueron obteniendo diferentes grupos de palabras representativas de las motivaciones de los informantes. Los resultados muestran un núcleo central de palabras común a todos los entrevistados, lo cual, según los autores, es muestra del elevado consenso existente entre los informantes.

\section{ANÁLISIS DE REDES TEXTUALES. LA CONCEPCIÓN DEL TEXTO COMO RED ARTICULADA DE PALABRAS Y DIFERENTES RELACIONES SEMÁNTICAS}

Las aproximaciones al análisis de textos que se han revisado en el epígrafe anterior toman de la teoría de redes sociales su fortaleza heurística, es decir la capacidad que esta perspectiva tiene de mostrar las informaciones de un modo revelador y sugerente desde el punto de vista analítico. Esta postura coincide con ciertos posicionamientos que consideran que la teoría de redes sociales no supone un modo particular de abordar la realidad social, sino simplemente una forma diferente de presentar la información: «es como un histograma, por ejemplo, que nos ayuda a dibujar la distribución de los datos pero que no proporciona capacidad analítica por él mismo» nos dice Freeman (1992:38).

Sin embargo, ciertos autores consideran que el análisis de redes sociales aporta al análisis de textos bastante más que un modo de representación de las palabras o conceptos presentes en un documento. Se trata, en estos casos, de reconocer y poner de manifiesto la naturaleza relacional de los textos: el sentido de un texto viene dado precisamente por la forma en que se relacionan las palabras que lo componen, y no sólo por las palabras en sí mismas. Dicho de otro modo, y utilizando el lenguaje propio de las redes sociales, el significado de un texto se obtiene poniendo el acento tanto en los nodos como en las relaciones que los vinculan. Esta perspectiva que se interesa por la naturaleza reticular de los textos la denominaremos aquí análisis de redes textuales, término que pretende ser una traducción del término inglés network text analysis, que podría traducirse también como «análisis de textos mediante redes».

Según Popping (2000:30), «el análisis de redes textuales [network text analysis] tuvo su origen con la observación de que después de que uno haya codificado las relaciones semánticas existentes entre conceptos, puede proceder a la construcción de redes de conceptos semánticamente vinculados». Como se comprueba, los ecos de las redes semánticas son evidentes. El análisis de redes textuales supone trasladar los principios relacionales aplicados en los mapas cognitivos o redes semánticas al análisis de textos. Se trata de procedimientos que no sólo suponen una identificación de las palabras o conceptos principales en un de- 
terminado texto, sino también la codificación de las relaciones entre estas palabras o conceptos - es decir una interpretación de los enunciados del texto-que después se agregan formando una red. Ello supone que la red finalmente obtenida - y que representa a todo el texto o conjunto de textos analizados - tiene una unidad semántica, y no es simplemente la suma de diferentes subredes de relaciones.

Las aproximaciones que encajan en estas premisas no son muy numerosas, aunque algunas llevan desarrollándose hace ya bastantes años. Dos características generales son compartidas en todas ellas: a) la necesidad de una interpretación (humana) del texto, puesto que el tipo de relación que une los términos de la red textual debe ser inferida a partir de la comprensión y primer análisis de los datos; y b) todas las representaciones mediante redes suponen una reducción (teóricamente guiada) del texto, puesto que recogen únicamente las relaciones que son de interés para el analista. Estas relaciones, así como los procedimientos específicos de codificación y representación, pueden ser muy variados, puesto que obedecen a los diferentes objetivos teóricos e intereses metodológicos de los autores. Dada esta disparidad de aproximaciones, estas propuestas de análisis se presentan a continuación en cuatro secciones diferentes, agrupadas por afinidades.

\subsection{Análisis reticular de textos de evaluación: Redes para encontrar las valoraciones implícitas}

El objetivo del análisis reticular de textos de evaluación (network analysis of evaluative texts) es la construcción de redes basadas en los enunciados explícitos en un texto, de modo que pueda llegarse a «la formulación de reglas de inferencia que revelan el contenido implícito o latente» (Kleinnijenhuis, de Ridder y Rietberg 1997:191). Esta aproximación fue originariamente desarrollada por Van Cuilenburg, Kleinnijenhuis y de Ridder (1988) como alternativa al análisis de contenidos totalmente automatizado, de modo que el software -el que ellos desarrollaron se denomina CETA - constituyese únicamente una ayuda en el análisis llevado a cabo por el/la analista.

El procedimiento propuesto por estos autores consiste en codificar los enunciados que se desean analizar en forma de relación valorada entre un sujeto y un objeto (puntuación entre -1 y 1 ). Pueden establecerse hasta quince tipos diferentes de conexión, que en el programa quedan simplificadas en cuatro tipos principales de vínculos: similitud, causalidad, relación emocional y asociación, con sus respectivas puntuaciones entre -1 y 1 . Una vez realizada esta codificación el programa CETA ofrece dos tipos de información: «sobre los juicios de valor realizados en torno a los objetos con significación en el texto [es decir, se excluyen artículos, adverbios, etc., y sobre las relaciones de evaluación entre esos objetos» (Van Cuilenburg, Kleinnijenhuis y de Ridder 1988:92). Las evaluaciones de carácter implícito se obtienen mediante el análisis de los paths existentes entre objetos en la red agregada.

EMPIRIA. Revista de Metodología de Ciencias Sociales. N. ${ }^{\circ}$ 10, julio-diciembre, 2005, pp. 129-150. 
Este procedimiento de análisis ha sido aplicado por los autores a diferentes tipos de textos. En el artículo publicado en 1988 (Van Cuilenburg, Kleinnijenhuis y de Ridder 1988) los datos analizados son enunciados creados ex profeso que recogen el estado de opinión existente en los medios de comunicación en relación al conflicto entre Irán y los Estados Unidos tras la revolución de Jomeini. En el trabajo publicado en 1997 (Kleinnijenhuis, de Ridder y Rietberg 1997) se analiza una muestra de los enunciados de las noticias de carácter económico aparecidas en dos periódicos holandeses entre 1968 y 1984. Tanto en un caso como en otro, la red representa las valoraciones implícitas presentes en los enunciados. El análisis permite descubrir, en el primer caso, una evaluación positiva de la relación entre Iraq y el sha, y en el segundo, el cambio de perspectiva producido a lo largo del tiempo en la presentación de la realidad económica por parte de la prensa.

\subsection{Map analysis: La disposición reticular de los conceptos}

Posiblemente Kathleen Carley y sus colaboradores constituyen el núcleo de autores que mayor producción han realizado en torno al análisis de redes textuales hasta el día de hoy. Actualmente, como directora del Center for Computational Analysis of Social and Organizational Systems (CASOS), Carley trabaja, entre otros temas, en la aplicación de la teoría de redes sociales al análisis de textos, habiendo desarrollado para ello el programa informático AutoMap. Esta autora denomina Map Analysis al tipo de procedimiento de análisis que utiliza. El objetivo de esta perspectiva es realizar un análisis formalizado de textos que permita «examinar procesos de decisión, modelos mentales, definiciones, estructuras conceptuales y el papel que juega el conocimiento social en ellos» (Carley 1993:108).

El Map Analysis trata de confeccionar una red textual -en un inicio con el soporte del programa MECA y en la actualidad con AutoMap- a partir de la codificación de los enunciados que aparecen en un texto o conjunto de textos. Para ello Carley distingue (1993: 92) entre los términos concepto, relación y enunciado (statement). Entre los primeros existen diferentes niveles de abstracción; por ejemplo, un nombre propio puede formar parte del concepto más general «gente». El segundo término, relación que puede diferir en intensidad, signo, dirección y significado lo entiende como el vínculo que existe entre dos conceptos; mientras que un enunciado consiste en dos conceptos más la relación que los une. La red textual que se obtiene con el análisis está constituida por todo el conjunto de enunciados codificados en un texto. Inicialmente pueden codificarse los enunciados que aparecen explícitamente, pero es posible que el/la analista pueda incorporar conceptos y relaciones (por lo tanto nuevos enunciados) que aparecen de forma implícita. El/la analista los incorpora gracias al conocimiento «contextual» y «social» de que dispone en relación a tema que está tratando (Carley 1997; Palmquist, Carley y Dale 1997). 
Los trabajos de esta autora se han desarrollado en múltiples direcciones, yendo desde planteamientos de análisis muy cercanos a intereses cognitivos (Carley y Palmquist 1992), hasta la representación de la estructura organizacional de redes ocultas en base al análisis documental (Diesner y Carley 2004), pasando por propuestas de análisis secuencial (Carley 1993, 1997). Si en sus primeros trabajos el objetivo principal era obtener el modelo mental subyacente mediante el análisis textual de unos pocos documentos, en sus últimas publicaciones el corpus de documentos analizados supera los varios cientos (Diesner y Carley 2004, 2005). En todos los casos, el tipo de grafos obtenidos son siempre dirigidos, aunque sólo los que analizan corpus pequeños son también valorados y contienen diferentes tipos de relación. Las medidas utilizadas varían en función de cada pregunta de investigación, aunque suelen coincidir con el grado nodal de salida, el grado nodal de entrada, el grado nodal total, la capacidad de intermediación y la capacidad de presencia en las relaciones (1997:85-6).

\subsection{Grafos de conocimiento: representando el conocimiento científico}

En cierto modo, el trabajo de Popping (2003) también tiene su origen en el deseo de aplicar los instrumentos desarrollados en la ciencia cognitiva al análisis de textos. Partiendo de los objetivos generales de las redes semánticas - es decir la representación estructurada del conocimiento- el autor pretende realizar un análisis de textos que sirva para representar el conocimiento de carácter científico compartido en un determinado campo. Para ello utiliza lo que denomina grafos de conocimiento (knowledge graphs), que además de estar basados en la explotación de información textual, se diferencian de las redes semánticas, según el autor, en «la elección explícita de sólo unos cuantos tipos de relación» (2003:93).

La primera etapa del procedimiento propuesto consiste en la codificación del conocimiento autor/a por autor/a. El conocimiento científico expresado individualmente es representado mediante un grafo de autor consistente en un conjunto de conceptos (nodos) vinculados entre ellos por diferentes tipos de relación, la más importante de las cuales es la relación de causalidad. Según afirma Popping, el objetivo inicial era el de representar el conocimiento utilizando principalmente la relación «ser causa de» aunque finalmente se han introducido una mayor variedad de relaciones. Una vez realizados estos grafos individuales comienza el proceso de agregación, mediante la comparación e integración (si se trata del mismo concepto o la misma relación) de cada una de las redes iniciales. Este proceso de agregación da lugar al grafo integrado, que debería representar el conocimiento compartido entre los diferentes autores/as analizados/as y, por lo tanto, en cierto modo, común dentro de la disciplina objeto de estudio.

Popping describe como la primera aplicación práctica de estas propuestas - a un conjunto de 6 tesis doctorales en torno a teorías sobre el mercado de trabajo- no fue exitosa, puesto que no se encontraron los suficientes conceptos 


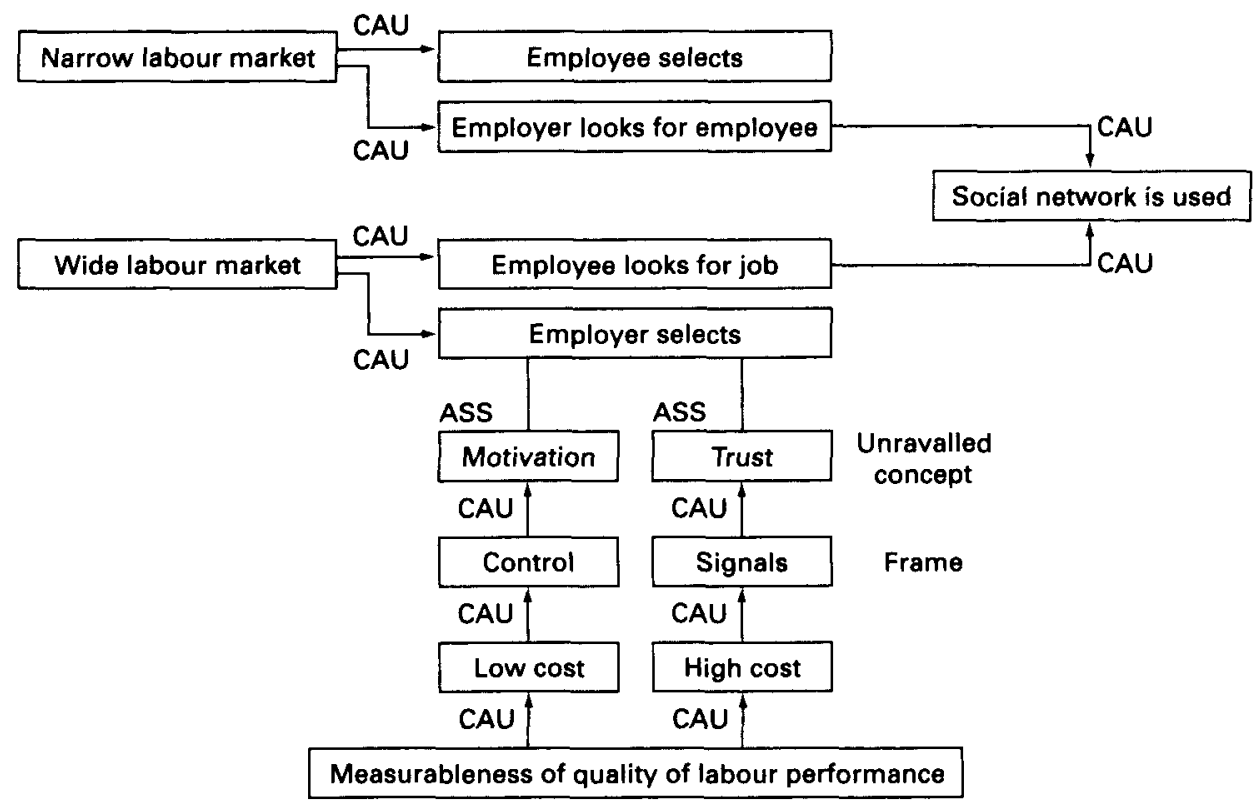

Fuente: Figura 4. Popping (2003: 103).

Figura 4. Grafo de conocimiento representando el funcionamiento de los mercados de trabajo según las teorías del control.

compartidos entre los/as diferentes autores/as. Posteriormente, el mismo procedimiento fue aplicado a un conjunto de ideas teóricas, no correspondientes a ninguna investigación específica. Concretamente, la red textual obtenida mediante el análisis pretendía representar las explicaciones sobre el funcionamiento del mercado de trabajo según las teorías del control (Popping 2003:102). La representación obtenida puede observarse en la Figura 4. Es relevante en esta red textual el intento por parte del autor tanto de descomponer los conceptos complejos (unravell concepts) como de poner de manifiesto cuáles son las ideas teóricas (frames) que se sitúan en el trasfondo de estos conceptos complejos.

\subsection{Análisis reticular del discurso: La preocupación por la interpretación contextualizada}

$\mathrm{Al}$ igual que los tres anteriores conjuntos de aproximaciones, el análisis reticular del discurso (ARD) pretende traducir a una red de relaciones un conjunto concreto de enunciados, caracterizando diferentes tipos de relaciones y tomando el conjunto del texto como unidad de análisis. Pero a diferencia de las anteriores, su planteamiento se aleja de los propósitos habituales en el análisis de 
contenido -en el sentido de búsqueda específica de unos determinados temas o contenidos - para acercarse a la concepción holista habitual en el análisis del discurso - entendiendo discurso como los contenidos superiores a la frase o proposición dentro de un texto-. De hecho el ARD surgió como un procedimiento aplicado al análisis de entrevistas, uno de cuyos objetivos principales era el de utilizar el máximo de información contextual para la codificación y análisis de los textos. Otra de sus características es la codificación de todos y cada uno de los enunciados del texto, con ello se pretenden minimizar las posibles pérdidas de información utilizando las mínimas unidades de registro con sentido. Todo ello busca potenciar la vertiente interpretativa del análisis, de modo que la reconstrucción del texto en forma de red sea una vía para mantener el carácter unitario original.

El ARD ha sido desarrollando de forma colectiva (Lozares, Martí y Verd 1997) en el seno del Departamento de Sociología de la Universidad Autónoma de Barcelona, aunque en sus aplicaciones prácticas pueden detectarse algunos elementos diferenciales en función de las preocupaciones y objetivos teóricos a que ha sido aplicado. Así, no es posible hablar de un único procedimiento, por lo que se señalan a continuación las características más destacadas de cada una de sus configuraciones?

Dentro de estas variaciones puede citarse en primer lugar una versión (Lozares 2000) interesada principalmente en las diferentes secuencias de episodios o situaciones de interacción que son descritas de forma narrativa. Esta aproximación considera que una narración puede contemplarse como un conjunto de entornos o dominios de interacción que van siendo (re)creados en el momento de la interlocución. De este modo el narrador presenta diferentes identidades del «yo», pero también diferentes contextos espaciales, temporales, objetuales y relacionales, que son vinculados mediante expresiones verbales de carácter diverso. Son estas expresiones verbales las que constituyen el contenido relacional de la red, siendo los diferentes objetos representados (no es su sentido de reproducidos tal como ocurrieron en el pasado, sino actualizados en función del contex to concreto de la entrevista) los que constituyen los nodos.

Una segunda versión (Martí 2000) de esta misma perspectiva se interesa por los aspectos argumentativos del discurso. Siguiendo la teoría de la argumentación de la lengua (Anscombre y Ducrot 1994), el procedimiento intenta identificar diferentes estructuras discursivas. Ello se hace mediante la codificación de relaciones tópicas (relaciones entre conceptos socialmente compartidas) al nivel del discurso y representándolas mediante la formalización reticular. El tipo de relación es dirigida y valorada. Una vez establecidas las relaciones se utiliza el álgebra de matrices para su análisis; en este análisis, indicadores tales como el grado nodal de salida y de entrada, así como indicadores de cercanía (closeness) e

7 Una descripción más detallada de las diferentes variaciones del ARD puede hallarse en Lozares (2000), Martí (2000), Verd y Lozares (2000), Verd (2002) y Lozares, Verd, Martí y López (2003).

EMPIRIA. Revista de Metodología de Ciencias Sociales. N. ${ }^{\circ}$ 10, julio-diciembre, 2005, pp. 129-150. 
intermediación (betweeness) indican los vínculos establecidos entre enunciados en el discurso, de modo que es posible conocer el mapa cognitivo que cada entrevistado posee en torno a los temas tratados.

Finalmente, una tercera versión (Verd y Lozares 2000; Verd 2002) se interesa por la reconstrucción de secuencias biográficas a partir de entrevistas narrativas. La red textual resultado del análisis se construye fundamentalmente a partir de relaciones de causalidad y de identidad, correspondiendo los nodos a acontecimientos o «hechos» que forman parte de la biografía de las personas entrevistadas. En este sentido, en relación con los otros dos desarrollos ya mencionados, es el que mayor atención presta a los elementos factuales del discurso. Aquí, la representación reticular se desarrollan en una doble dimensión, por un lado mediante jerarquías de conceptos, mostrando la dimensión cognitiva de los enunciados del narrador, y por otro lado, mediante relaciones de causalidad vinculando en forma de secuencia los acontecimientos descritos en la narración. Podría decirse, por lo tanto, que es la propuesta más próxima a ciertos enfoques que intentan formalizar el contenido causal de las narraciones (Abell 1987), aunque intentando introducir en el análisis una aproximación que procura acceder a las construcciones que realiza el propio actor.

\section{ALGUNOS ELEMENTOS DE CONCLUSIÓN}

A lo largo del artículo se ha querido mostrar en qué medida y de qué modo la perspectiva de redes sociales resulta de utilidad en el análisis de textos. Como se ha puesto de manifiesto, los usos actuales del análisis de redes sociales en este campo pueden entroncarse con las representaciones mediante grafos de aplicación en las ciencias cognitivas. Esta vinculación es especialmente evidente en aquellos procedimientos que hemos englobado bajo el término análisis de redes textuales - en algunos casos la hacen explícita los propios autores que han desarrollado los procedimientos de análisis- puesto que la pretensión no es sólo presentar los vínculos entre los conceptos o términos principales que aparecen en un texto o conjunto de textos, sino también mostrar qué tipo de relaciones semánticas unen estos conceptos o términos principales.

El artículo ha pretendido mostrar, también, los dos grandes grupos en que se pueden organizar los procedimientos de análisis que utilizan la perspectiva de redes sociales. Por un lado nos encontramos con todo un conjunto de trabajos en que la relación representada en las redes es la «copresencia» de términos o palabras, mientras que por otro lado existen un número relativamente limitado de procedimientos que pretenden dotar a las relaciones representadas de significados más complejos y a su vez más ricos analíticamente. De hecho, son este segundo bloque de procedimientos los que llevan al máximo las posibilidades que ofrece el análisis de redes sociales como herramienta de investigación. Son también estos procedimientos los que más se alejan de los análisis de contenidos o temáticos de orientación cuantitativa. Estos procedimientos que aplican en pro- 
fundidad la teoría de redes sociales parten de la premisa básica de que es más coherente con la naturaleza articulada de un texto y más eficaz en la búsqueda de su sentido el respetar las relaciones que se establecen entre sus elementos o componentes. Como defiende Carley (1993), la posibilidad de codificar no tan solo los conceptos (en la forma del análisis de contenido tradicional) sino también las relaciones entre ellos permite una mejor interpretación de los textos. Utilizando un símil pictórico... dos paisajes pueden resultar absolutamente diferentes a pesar de estar compuestos exactamente por los mismos elementos; lo que marcará la diferencia es la interrelación entre esos distintos elementos.

Un punto destacado que marca una de las diferencias fundamentales entre lo que hemos denominado análisis de redes de palabras y análisis de redes textuales es el grado de intervención humana en el proceso de análisis. Una de las características definitorias del análisis de redes de palabras es el uso de la codificación automatizada mediante ordenador.

Las propuestas que hemos denominado globalmente análisis de redes textuales también suelen apoyarse en el uso de algún programa informático, pero la codificación es siempre en última instancia humana. Sólo estas propuestas, que tienen una aproximación más marcadamente interpretativa, superan los problemas provenientes de la correcta comprensión del significado de los enunciados. La presencia de un codificador humano permite resolver las ambigüedades semánticas del texto mediante la utilización de conocimiento contextual. Es verdad que ello exige una mayor inversión temporal en la codificación ${ }^{8}$, pero tiene poco sentido dejar de lado precisamente aquello que supone una fortaleza en la aplicación de la perspectiva de redes sociales, que no es más que su capacidad para mantener la estructura de significados, frente a los análisis que la fragmentan.

Con todo, tanto el análisis de redes de palabras como el análisis de redes textuales suponen una superación de los análisis temáticos clásicos, tanto cuantitativos como cualitativos. Cuando se representa en forma de red un texto o un discurso se están ubicando unos determinados conceptos o enunciados en relación al resto, se están describiendo distancias, recorridos... en definitiva, se da un sentido unitario al conjunto. En los análisis temáticos o de contenidos este sentido unitario se consigue una vez realizado el análisis, en los que utilizan la perspectiva de redes sociales es un elemento intrínseco al propio procedimiento de análisis.

Para finalizar, es interesante mencionar la evolución que pueden adoptar en el futuro estos procedimientos que hemos revisado. Por un lado es previsible un mayor desarrollo de programas informáticos inspirados en estos procedimientos, especialmente de los vinculados al análisis de redes textuales, puesto

${ }^{8}$ Esto es especialmente cierto en el análisis reticular del discurso, puesto que es también el que manifiesta mayor insistencia en estos procesos de interpretación contextualizada. Este procedimiento pone gran énfasis en la relación entre los contextos internos y externos del discurso, cuando en general la codificación tiende a recoger principalmente el contexto intratextual. 
que son los que a día de hoy se encuentran aún sin versiones plenamente operativas. Al respecto puede visitarse la página web del Center for Computational Analysis of Social and Organizational Systems (http://www.casos.cs.cmu.edu) en que se está desarrollado el programa AutoMap. Los programas orientados hacia el análisis de redes de palabras están algo más desarrollados, alguno de ellos con años ya de comercialización. Pueden consultarse a este respecto las páginas web del Laboratory for Organization, Communication and Knowlege Studies (http://www.locks.asu.edu) y de la empresa Megaputer (http://www.megaputer.com).

Por otra parte es probable que surjan procedimientos estándar -posiblemente vinculados a los propios programas informáticos mencionados anteriormente- que establezcan vías para la reducción del tamaño de las redes. Precisamente la magnitud de las redes que se analizan es uno de los principales problemas con los que se enfrenta la aplicación de la teoría de redes sociales al análisis de textos. En general, tanto los análisis que construyen redes de palabras como los que construyen redes textuales producen redes con cientos de nodos. Ello no supone un problema para su tratamiento mediante los programas de análisis de redes existentes en el mercado (véase al respecto Batagelj, Mrvar y Zaveršnik 2002), pero sí dificulta la presentación gráfica de los datos, e incluso su interpretación teórica por parte del/de la analista. Ello ha originado numerosas propuestas encaminadas a la reducción del tamaño de las redes, que básicamente pueden sintetizarse en dos orientaciones: aquellas que reducen el número de nodos (véase Corman, Kuhn, Mcphee, y Dooley 2002:175; Diesner y Carley 2004) y aquellas que reducen el número de relaciones (véase Borgatti 1997; Popping 2000:109-115). Las primeras son las más interesantes, puesto que en general la densidad de este tipo de redes no es elevada, con lo que la reducción de relaciones no resulta una prioridad.

Una vez más, es recomendable la intervención humana en los procesos de reducción de redes, tanto si se han contruido mediante los procedimientos del análisis de redes de palabras como si se han construido mediante procedimientos englobables en el análisis de redes textuales. Como se comprueba, pues, la aplicación de la teoría de redes sociales al análisis de textos permite favorece la realización de análisis más rigurosos, transparentes y con un mayor grado de formalización, pero por el momento no exime al investigador/a social del trabajo de interpretar, categorizar y dotar de sentido teórico a las redes analizadas. ¡Habrá que seguir buscando! 


\section{BIBLIOGRAFIA}

ABell, P. (1987): The Syntax of Social Life. Oxford, Clarendon Press.

ANSCOMBRE, J. C. y DUCROT, O. (1994) La argumentación en la lengua. Madrid, Gredos.

BATAGEl, V.; MRVAR, A. y ZAVERŠNIK, M. (2002): «Network Analysis of Texts». En: T. Erjavec y J. Gros (eds.): Proceedings of the $5^{\text {th }}$ International Multi-Conference Information Society - Language Technologies/Jezikovne technologije, Ljubljana.

BAuER, M. W. (2000): «Classical Content Analysis: a Review». En: Martin W. Bauer y George Gaskell (eds.): Qualitative Researching with Text, Image and Sound. Londres, Sage.

BRANDES, U.; CORMAN, S. R. (2003) «Visual unrolling of network evolution and the analysis of dynamic discourse». Information Visualization, 2(1):40-50.

Borgatri, S. P. (1997): Causal Cognitive Networks. Comunicación presentada en el Qualitative Data Analysis Workshop, Universidad de Ginebra, julio de 1997.

CARLEY, K. (1993): «Coding choices for textual analysis: A comparison of content analysis and map analysis». Sociological Methodology, 23:75-126.

- (1997): «Network text analysis: The network position of concepts». En: Carl W. Roberts (ed.): Text Analysis for the Social Sciences: Methods for Drawing Statistical Inferences from Texts and Transcripts. Mahwah, New Jersey: Lawrence Erlbaum.

CARLEY, K.; PALmQuist, M. E. (1992): «Extracting, representing, and analyzing mental models». Social Forces, 70 (3): 601-636.

Van Cuilenburg, J. J.; KleinniJenhuis, J. y De Ridder, J. A. (1988): «Artificial intelligence and content analysis. Problems of and strategies for computer text analysis». Quality and Quantity, 22:65-97.

Corman, S. R. y Dooley, K. J. (2001): Centering Resonance Analysis of Reuters News of the September $11^{\text {th }}$ Attack, Days $l$ and 2. <http://www.locks.asu.edu/terror/cra reutersdemo_files/frame.htm>, documento activo el 01/09/2005.

Corman, S. R.; Kuhn, T.; McPheE, R. D. y Dooley, K. J., (2002): «Studying complex discursive systems: Centering resonance analysis of communication». Human Communication Research, 28(2): 157-206.

DANOWSKI, J. A. (1988): «Organizational Infographics and Automated Auditing: Using Computers to Unobstrusively Gather as Well as Analyze Communication». En: G. M. Goldhaber y G. A. Barnett (ed.): Handbook of Organizational Communication. Norwood, New Jersey: Ablex.

- (1993): «Network Analysis of Message Content». Progress in Communication Sciences, 12:198-221.

Diesner, J.; CARLEY, K. M. (2004): «Using Network Text Analysis to Detect the Organizational Structure of Covert Networks". En: Proceedings of the North American Association for Computational Social and Organizational Science (NAACSOS) 2004 Conference, Pittsburgh.

- (2005): Revealing and Comparing the Organizational Structure of Covert Networks with Network Text Analysis. Comunicación presentada en la XXV Sunbelt Social Network Conference, Redondo Beach, California, febrero de 2005.

Doerfel, M. L. (1998): «What Constitutes Semantic Network Analysis? A Comparison of Research and Methodologies». Connections, 21(2):16-26.

FreEMAN, L. (1992): «Social Networks and the Structure Experiment». En: L. C. Freeman, D. R. White y A. K. Romney (ed.): Research Methods in Social Network Analysis. Londres: Transaction Publishers. 
Freeman, C. A.; BarnetT, G. A. (1994): “An Alternative Approach to Using Interpretative Theory to Examine Corporate Messages and Organizational Culture». En: L. Thayer y G. A. Barnett (ed.): Organization Communication. Emerging Perspectives. $I V$. Norwood, New Jersey: Ablex.

GlaSER, B. G.; STRauss, A. L. (1967): The Discovery of Grounded Theory. Strategies for Qualitative Research. Nueva York: Aldine.

JANG, H. y BARNETT, G. A. (1994): «Cultural Differences in Organizational Communication: A Semantic Network Analysis». Bulletin de Methodologie Sociologique, 44:31-59.

JOHNSON-LAIRD, P. N. (1990) [1988]: El ordenador y la mente. Introducción a la ciencia cognitiva. Barcelona, Paidós.

KleINNIJENhUIS, J.; De RIDDER, J. A. y RietberG, E. M. (1997): «Reasoning in Economic Discourse: An Application of the Network Approach to the Dutch Press», en Carl W. Roberts (ed.): Text Analysis for the Social Sciences: Methods for Drawing Statistical Inferences from Texts and Transcripts. Mahwah, New Jersey: Lawrence Erlbaum.

KRIPPENDORF, K. (2004): Content Analysis. An Introduction to lts Methodology. Thousand Oaks (Calif.): Sage.

Lonkila, M.; HaRmo, T. (1999): «Toward Computer-Assisted Qualitative Network Analysis». Connections, 22(1):52-61.

LOZARES, C. (2000): «El discurs reticular, més enllà de la classificació». Revista Catalana de Sociologia, 11:183-9.

LOZARES, C.; MARTí, J. y VERD, J. M. (1997): Análisis reticular del discurso. Universitat Autònoma de Barcelona, Departament de Sociologia. Texto no publicado.

LOZARES, C.; VERD, J. M.; MARTI, J; LÓPEZ, P. (2003): «Relaciones, redes y discurso: revisión y propuestas en torno al análisis reticular de datos textuales». Revista Española de Investigaciones Sociológicas, 101:175-200.

MARTI, J. (2000): Formació i ocupació en el discurs dels treballadors. Una proposta metodològica. Universitat Autònoma de Barcelona, Departament de Sociologia. Tesis Doctoral.

VAN METER, K. M. (1999): «Social Capital Research Literature: Analysis of Keyword Content Structure and the Comparative Contribution of Author Names». Connections, 22(1):62-84.

VAN METER, K. M. y TURNer, W. A. (1997): «Representation and Confrontation of Three Types of Longitudinal Network Data from the same Data Base on Sociological AIDS Research». Bulletin de Methodologie Sociologique, 56:32-49.

Miles, M. B. y Huberman, A. M. (1994): Qualitative Data Analysis. An Expanded Sourcebook. Thousand Oaks, California, Sage.

MuHR, T.; FrIESE, S. (2004): User's Manual for ATLAS.ti 5.0. Berlin, Scientific Software Development. 2. ${ }^{\mathrm{a}}$ edición.

PALMQUist, M. E.; CARLEY, K. M. y Dale, T. A. (1997): «Applications of computer-aided text analysis: Analyzing literary and nonliterary texts». En: Carl W. Roberts (ed.): Text Analysis for the Social Sciences: Methods for Drawing Statistical Inferences from Texts and Transcripts. Mahwah, New Jersey: Lawrence Erlbaum.

PoPPING, R. (2000): Computer-assisted Text Analysis. London: Sage.

- (2003): «Knowledge graphs and network text analysis». Social Science Information, 42:91-106.

PREIN, G.; Kelle, U.; RICHARDS, L. y RICHARDS, T. (1995): «Introduction: Using linkages and networks for qualitative theory building», en Udo Kelle (ed.): Computer-aided Qualitative Data Analysis. Londres, Sage. 
RICHARDS, T. J. y RICHARDS, L. (1994) «Using Computers in Qualitative Research». En: Norman K. Denzin e Yvonna S. Lincoln (ed.): Handbook of Qualitative Research. Thousand Oaks, California, Sage.

SCHNEGG, M. (1997): «Words as Actors II: Semantic Communities and their Overlap». Comunicación presentada en el Qualitative Data Analysis Workshop, Universidad de Ginebra, julio de 1997.

SchnegG, M. y Bernard, H. R. (1996): «Words as Actors: A Method for Doing Semantic Network Analysis». Cultural Anthropology Methods Journal, 8 (2): 7-10.

SowA, J. F. (1991): «Current Issues in Semantic Networks». En: John F. Sowa (ed.): Principles of Semantic Networks. Explorations in the Representation of Knowledge. San Mateo, California: Morgan Kaufman.

VERD, J, M. (2002): Itinerario biográfico, recursos formativos y empleo. Una aproximación integrada de carácter teórico y metodológico. Universitat Autònoma de Barcelona, Departament de Sociologia. Tesis Doctoral. (Puede consultarse el texto en http://www.tdr.cesca.es/index_tdx_cs.html)

VERD, J. M. y LOZARES, C. (2000): «La teoria de xarxes socials aplicada a la interpretació d'entrevistes narratives. Una proposta». Revista Catalana de Sociologia, 11:1916.

WUEHReR, G. A. (2001): «Cognitive Maps of Export Marketing Strategies. Preliminary Results from a Quantitative and Qualitative Study». En: S. Grabner-Kraeter y A. G. Wuehrer (eds.): Trends im Internationalen Management. Strategien, Instrumente und Methoden. Festschrift für Profesor Dr. Dieter J. G. Schneider. Linz: Trauner Universitaetsverlag. 


\section{RESUMEN}

El artículo revisa el conjunto de aproximaciones al análisis de textos que se han inspirado en la teoría de redes sociales. Estas aproximaciones se agrupan en torno a dos orientaciones principales: aquellas que buscan fundamentalmente la representación y análisis de las palabras presentes en un texto dejando en una posición secundaria el tipo de relación que las une, y aquellas que otorgan la misma importancia tanto a las palabras que conforman el texto como al tipo de relaciones que las vinculan y les dan sentido semántico. El primer tipo de aproximación, que se ha denominado análisis de redes de palabras, es el más extendido, y presenta algunos inconvenientes que el segundo tipo de análisis -que se ha denominado análisis de redes textuales-intenta resolver.

\section{PALABRAS ClAVE}

Análisis de textos, Análisis de redes textuales, Análisis de redes de palabras, Redes semánticas, Análisis de redes sociales.

\section{ABSTRACT}

The article reviews the group of approaches to text analysis that have been inspired by social network theory. These approaches are set in two main orientations: those that search basically the representation and analysis of words that are present in a text, leaving in a secondary place the type of relation that links them, and those that give the same importance both to words in the text and relations that bond words and provide semantic meaning. The first kind of approach, that has been called word-network analysis and it is the most extended one, presents some drawbacks that the second kind of approach which has been called network text analysis tries to solve.

\section{KEY WORDS}

Text Analysis, Network Text Analysis, Word Network Analysis, Semantic Networks, Social Network Analysis. 